\title{
An Extremely Rare Case of Leukoderma Induced by Cosmetics Containing Ascorbic Acid
}

\author{
Madoka Takafuji Atsushi Tanemura Ichiro Katayama \\ Department of Dermatology, Osaka University Graduate School of Medicine, Suita, Japan
}

\section{Keywords}

Cosmetics-induced leukoderma $\cdot$ Hypopigmented macule $\cdot$ Ascorbic acid $\cdot$ Melanocyte stem cells

\begin{abstract}
We report the case of a 73-year-old woman with leukoderma induced by cosmetics containing ascorbic acid. She had noticed the sudden appearance of a hypopigmented macule on the cheek within 1 month after use of the cosmetics with the brand name Obaji C Serum. No history of use of rhododenol-containing cosmetics was declared. Histopathological analysis revealed that the number of matured melanocytes was apparently decreased, and T lymphocytes abundantly infiltrated mainly the perifollicular region. In addition to the discontinuation of using Obaji C Serum, phototherapy by excimer light was noticeably effective for repigmentation. To our knowledge, there has been no other report so far showing hypopigmented lesions induced by this cosmetic.

(C) 2019 The Author(s) Published by S. Karger AG, Basel
\end{abstract}

\section{Introduction}

Cosmetics-induced leukoderma is defined as an acquired hypopigmentation caused by repeated exposure to specific agents damaging to epidermal melanocytes. No absolute criteria can distinguish cosmetics-induced leukoderma from vitiligo vulgaris, an acquired chronic disorder characterized by skin depigmentation due to localized loss of epidermal melanocytes. 
We report a case of leukoderma induced by cosmetics containing ascorbic acid, which was successfully treated with phototherapy by excimer light. The cosmetics' brand name is Obaji C Serum. Obaji C Serum is a whitening cosmetic containing ascorbic acid and has been cumulatively sold 7.8 million times in Japan.

\section{Case Report}

We experienced an extremely rare case of leukoderma induced by cosmetics containing ascorbic acid. A 73-year-old Japanese woman noticed the sudden appearance of hypopigmented macules on the cheek within 1 month after starting to use a cosmetic agent (brand name Obaji C Serum). She had initially felt itching with slight erythema corresponding to the application site. She was admitted to our hospital because the irregularly shaped hypopigmented macules rapidly extended to her bilateral cheeks, forehead, and area around the lip 4 months after starting to use the cosmetics (Fig. 1a, b). She had a history of systemic sclerosis and was positive for anti-HCV antibody without any current medication. The results of a component patch test were negative, indicating no allergic reaction to the ingredients. In addition to stopping the use of this cosmetic, phototherapy using excimer light noticeably accelerated repigmentation of the right cheek compared to the untreated left side (Fig. 1c-e). Perilesional hyperpigmentation was naturally relieved after discontinuing phototherapy. A histopathological analysis of her right cheek lesion before treatment revealed slight inflammation and solar damage in the upper to middle dermis (Fig. 2a). The number of Melan $\mathrm{A}^{+}$matured melanocytes was apparently decreased not only in the lesional but also in the perilesional skin (Fig. 2b). $\mathrm{CD}^{+}$and $\mathrm{CD}^{+} \mathrm{T}$ cells had predominantly infiltrated around the hair follicle, similar to cases of rhododenol-induced (RD) leukoderma, which has recently been of public concern in Japan $[1,2]$ (Fig. 2c, d). RD leukoderma exerts melanocyte-specific toxicity and subsequent inflammation depending on tyrosinase activity [2]. The histopathological findings differed markedly from those of vitiligo [1].

To confirm whether immature melanoblasts or melanocyte stem cells (MSCs) remained in the hypopigmented region, we performed immunostaining for frizzled-4 (FZD4) and microphthalmia-associated transcription factor (MITF) in the hair follicle [3]. Since FZD4 ${ }^{+} \mathrm{MITF}^{+}$ cells were observed in the infundibulum, the FZD4+MITF+ MSCs may supply matured melanocytes to the epidermis and contribute to rapid repigmentation (Fig. 2e).

\section{Discussion/Conclusion}

We experienced an extremely rare case of leukoderma induced by cosmetics containing highly concentrated ascorbic acid. Obaji C Serum (C5, C10, and C20 series) is a popular whitening cosmetic that contains ascorbic acid and has been cumulatively sold to 7.8 million consumers in Japan since 2001. In contrast, RD leukoderma, one of the whitening cosmetics-induced leukodermas, was observed in 10,000 consumers [4] by various mechanisms [2]. To our knowledge, there have been no reports describing hypopigmented lesions induced by this cosmetic agent. In our case, the clinical course and histopathological analysis suggest that this cosmetic is indeed associated with melanocyte disappearance and the onset of leukoderma. Although the possibility of contact dermatitis was considered for leukoderma following an allergic reaction to the cosmetics as shown in rhododenol-induced leukoderma [5], it was denied because of the negative patch tests of all components in this case. The exact reason why 


\section{Case Reports in Dermatology}

Takafuji et al.: An Extremely Rare Case of Leukoderma Induced by Cosmetics Containing Ascorbic Acid

this cosmetic induced hypopigmentation is unclear, although ascorbic acid itself is known to inhibit melanin synthesis by reducing dopa without exerting cytotoxic effects on melanocytes [6]. In the present case, a higher concentration of this cosmetic than expected may have accidentally penetrated the hair follicle, consequently influencing the homeostasis of MSCs and melanocyte survival. Further ex vivo experiments of whitening agents by using a facial skin model with hair follicles will aid in our understanding of the molecular mechanisms underlying various types of cosmetics-induced leukoderma.

\section{Acknowledgement}

We greatly appreciate Profs. Suzuki and Okamura, Yamagata University Faculty of Medicine, for performing an immunohistochemical staining for MSC.

\section{Statement of Ethics}

Informed consent was obtained from the patient. The study complied with the Declaration of Helsinki.

\section{Disclosure Statement}

The authors declare no conflict of interest.

\section{Author Contributions}

We all are responsible for and agree with the content and writing of the manuscript to which we all contributed significantly.

\section{References}

1 Tanemura A, Yang L, Yang F, Nagata Y, Wataya-Kaneda M, Fukai K, et al. An immune pathological and ultrastructural skin analysis for rhododenol-induced leukoderma patients. J Dermatol Sci. 2015 Mar;77(3):185-8.

2 Sasaki M, Kondo M, Sato K, Umeda M, Kawabata K, Takahashi Y, et al. Rhododendrol, a depigmentationinducing phenolic compound, exerts melanocyte cytotoxicity via a tyrosinase-dependent mechanism. Pigment Cell Melanoma Res. 2014 Sep;27(5):754-63.

3 Okamura K, Ohe R, Abe Y, Ueki M, Hozumi Y, Tamiya G, et al. Immunohistopathological analysis of frizzled-4positive immature melanocytes from hair follicles of patients with Rhododenol-induced leukoderma. J Dermatol Sci. 2015 Nov;80(2):156-8.

4 Nishigori C, Aoyama Y, Ito A, Suzuki K, Suzuki T, Tanemura A, et al. Guide for medical professionals (i.e., dermatologists) for the management of Rhododenol-induced leukoderma. J Dermatol. 2015 Feb;42(2):11328.

5 Aoyama Y, Ito A, Suzuki K, Suzuki T, Tanemura A, Nishigori C, et al. The first epidemiological report of rhododenol-induced leukoderma in Japan based on a nationwide survey. Jpn J Dermatol. 2014 Oct;124(11):2095-109.

6 Ros JR, Rodríguez-López JN, García-Cánovas F. Effect of L-ascorbic acid on the monophenolase activity of tyrosinase. Biochem J. 1993 Oct;295(Pt 1):309-12. 


\section{Case Reports in Dermatology}
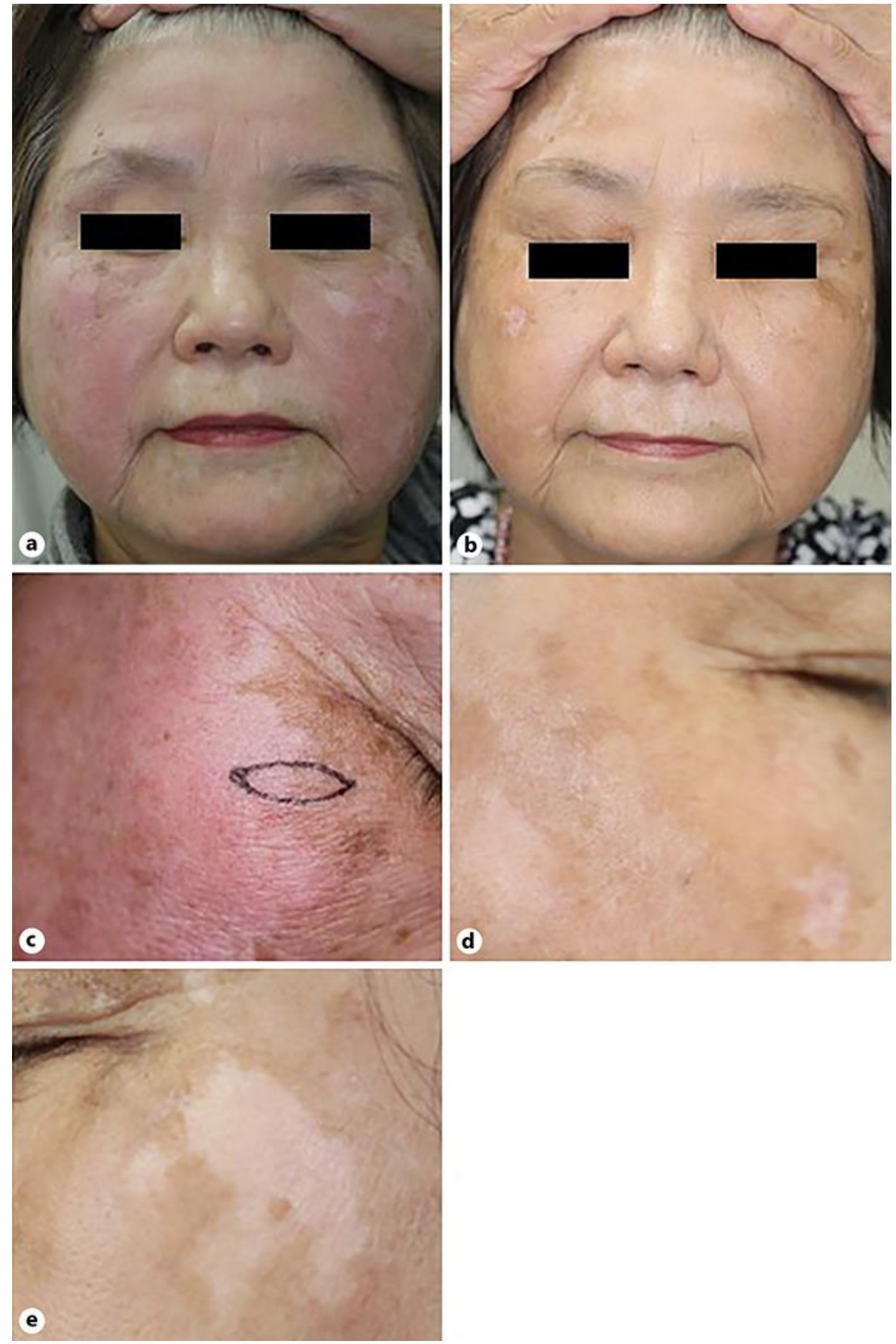

Takafuji et al.: An Extremely Rare Case of Leukoderma Induced by Cosmetics Containing Ascorbic Acid

Fig. 1. a Clinical features at the first visit. A number of leukoderma lesions were noted on the patient's face. b A biopsy specimen depicted by a spindle-shaped line was taken from the border of a leukoderma on her right cheek. c More abundant repigmentation was obtained on the right side treated with excimer light 10 months after the 1 st visit. d, e Close-up views of her right and left cheeks, respectively. 


\section{Case Reports in Dermatology}
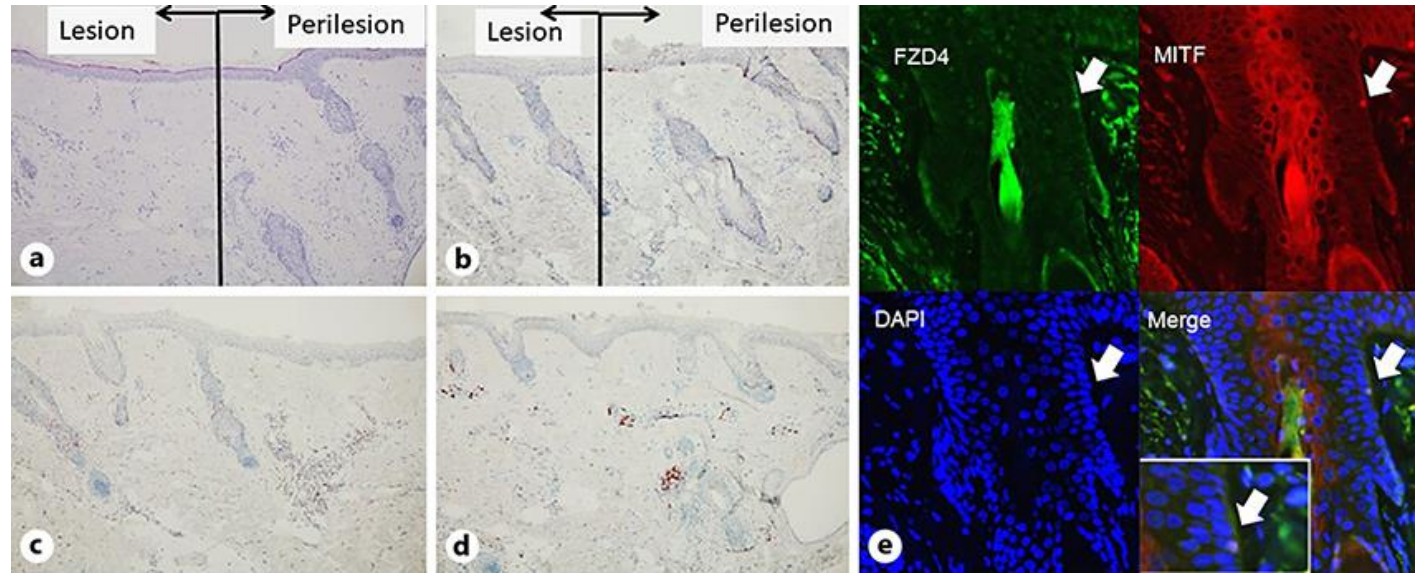

Fig. 2. a The histological findings showed melanocytes apparently decreased in number on not only the lesional but also the perilesional skin (HE staining, original magnification $\times 100$ ). $\mathbf{b}$ Immunohistochemical analysis showed no matured melanocytes positive for Melan A (×100). c, d CD4+ $(\times 100)$ (c) and CD8+ T cells $(\times 100)(d)$ infiltrated mainly the perifollicular regions, respectively. e Confocal microscopy analysis of cells expressing MITF (red) and FZD4 (green). Nuclei were stained blue with DAPI. MITF- and FZD4double positive cells (arrows) were observed in the infundibulum of the hair follicle. Inbox High-power magnification view. 